\title{
Hybrid Learning for Tool Wear Monitoring
}

\author{
X. Li $^{1,2}$, S. Dong ${ }^{2}$ and P. K. Venuvinod ${ }^{1}$ \\ ${ }^{1}$ Department of Manufacturing Engineering, City University of Hong Kong, Hong Kong; and ${ }^{2}$ Department of Mechanical Engineering, \\ Harbin Institute of Technology, Harbin, China
}

In automated manufacturing systems such as flexible manufacturing systems (FMSs), one of the most important issues is the detection of tool wear during the cutting process. This paper presents a hybrid learning method to map the relationship between the features of cutting vibration and the tool wear condition. The experimental results show that it can be used effectively to monitor the tool wear in drilling. First, a neural network model with fuzzy logic $(F N N)$, responding to learning algorithms, is presented. It has many advantageous features, compared to a backpropagation neural network, such as less computation. Secondly, the experimental results show that the frequency distribution of vibration changes as the tool wears, so the r.m.s. of the different frequency bands measured indicates the tool wear condition. Finally, FNN is used to describe the relationship between the characteristics of vibration and the tool wear condition. The experimental results demonstrate the feasibility of using vibration signals to monitor the drill wear condition.

Keywords: Fuzzy neural network; Vibration; Wear

\section{Introduction}

The development of an effective means to monitor the wear condition of cutting tools is one of the most important issues in the automation of the cutting process. The consequences of non-detection of tool failures may result in a poor quality product and damage to the workpiece or machine [1-5]. This paper focuses on monitoring of tool wear in drilling. Drilling is an important material removal process and it is therefore necessary to search for an effective way of monitoring drill wear.

The alternating direction of cutting force leads to vibrations of the machine structure. These vibrations change owing to the tool wear conditions. Despite the relatively harsh environment in the proximity of the cutting zone, the vibrations can

Correspondence and offprint requests to: Dr Xiaoli Li, Department of Manufacturing Engineering, City University of Hong Kong, 83 Tat Chee Avenue, Kowloon, Hong Kong. E-mail: mel50001@cityu.edu.hk be measured conveniently by accelerometers at a comparably affordable price.

Neural networks have been used for the classification of various signals for a long time. The most commonly used neural network in manufacturing related research uses a backpropagation (BP) algorithm. However, a BP requires a long learning time which limits its applicability. Hence, a neural network with fuzzy inference is used instead because of its ability to learn fast.

This paper proposes a new drill condition monitoring method based on a fuzzy neural network. Spectral analysis of the vibration signal is used to generate a set of indices for monitoring. The relationship between the tool wear condition and these indices is described by a fuzzy neural network. The results show that the approach is feasible.

\section{Fuzzy Neural Network}

\subsection{Combination of Fuzzy System and Neural Network}

Neural networks are organised in layers, each consisting of neurons or processing elements that are interconnected. There are a number of learning methods for training neural nets, but the back propagation (back-prop) paradigm has emerged as the most popular training mechanism. The back-prop method works by measuring the difference between output and the observed output value. The values at the output layer are propagated to the previous layers and used for adjusting the connection weights. However, there are potential drawbacks:

1. No clear guidelines on how to design neural nets.

2. The accuracy of results relies heavily on the size of the training set.

3. The logic behind the estimation is hard to convey to the user.

4. Long learning time.

5. Part convergence.

In order to overcome these drawbacks, a hybrid model of the neural network and fuzzy logic is presented. Although there are many possible combinations of the two systems, the 


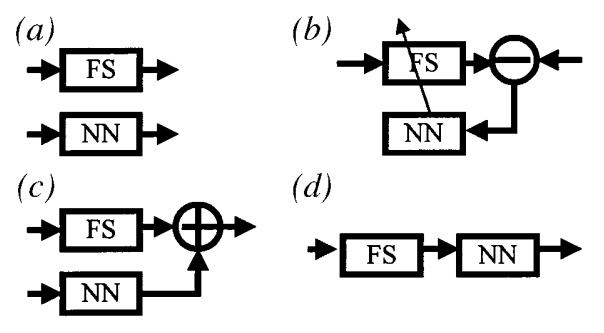

Fig. 1. Combination types of NN and FS.

four combinations shown in Fig. 1 have been applied to actual products.

Figure 1(a) shows the case where one piece of equipment uses the two systems for different purposes without mutual cooperation. The model in Fig. 1 $(b)$ uses the neural network (NN) to optimise the parameters of the fuzzy system (FS) by minimising the error between the output of the FS and the given specification. Figure 1(c) shows a model where the output of an FS is corrected by the output of an NN to increase the precision the final system output. Figure $1(d)$ shows a cascade combination of an FS and an NN where the output of the FS or NN becomes the input of another $\mathrm{NN}$ or FS. Figures $1(b)$ and $1(c)$ models refer to a combination model with net learning and a combination model with an equal structure, respectively. These are described in detail (see Fig. 2 ). Figure 2(a) shows that the total system is decribed by means of a fuzzy system, but the membership of the fuzzy system is produced and adjusted by the learning of the neural network. The model in Fig. 2(b) shows that a fuzzy system can be described by a neural network, the $\max (\bigvee)$ and min $(\wedge)$ operators for fuzzy inference are used to map the relationship between input and output of the FNN. The neural network is not a black box. The inference processing of the fuzzy system is responded to by the neural network. In the paper, a new neural network with fuzzy inference is presented. It has some features:

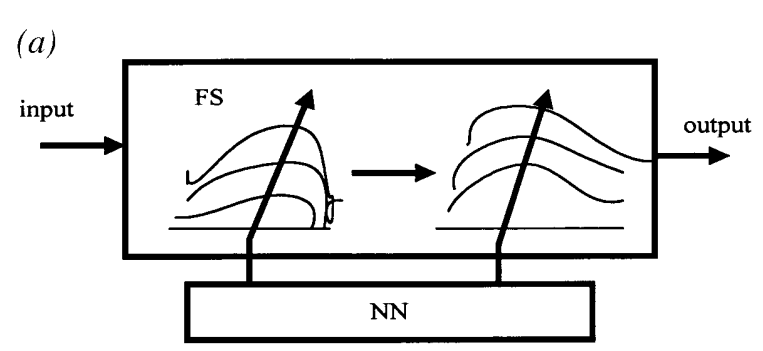

(b)

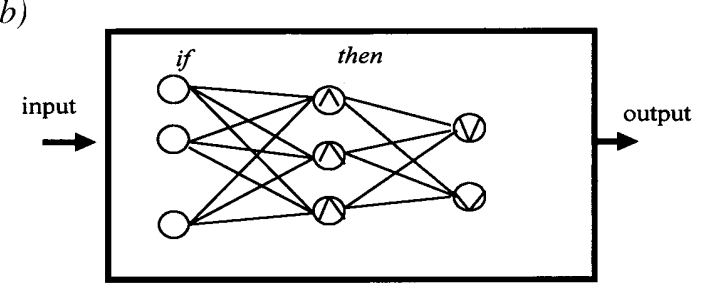

Fig. 2. (a) Combination model with net learning. (b) Combination model with equal structure.
1. Higher learning speed.

2. Better flexibility.

3. Convergence.

\subsection{FNN Net Topology and Learning Algorithms}

Suppose the input pairs are $\left(x_{1}, x_{2}, \ldots, x_{n}\right)$ and the output pairs are $\left(y_{1}, y_{2}, \ldots, y_{m}\right)$, and $y_{j}$ is determined by $x_{i}$ and $w_{i j}$. It is defined as follows:

$$
y_{j}=\max \left(\min \left(x_{i}, w_{i j}\right)\right)
$$

where $w_{i j}$ are the elements of the weight matrix $W$. The FNN net topology is shown in Fig. 3. Suppose the neural network has no hidden layer, if $X=\left(x_{1}, x_{2}, \ldots, x_{n}\right), Y=\left(y_{1}, y_{2}, \ldots\right.$, $\left.y_{m}\right)$ and $W=\left(w_{i j}\right), Y$ is obtained such that

$$
Y=X \circ W
$$

and

$$
y_{j}=\max \left(\min \left(x_{i}, w_{i j}\right)\right) \quad(i=1,2, \ldots, n ; j=1,2, \ldots, m)
$$

where $X \in[0,1], Y \in[0,1]$. Let $X$ and $Y$ be the input data $\left(x_{1}, x_{2}, \ldots, x_{i}, \ldots, x_{n}\right)$ and the desired output value $\left(y_{1}, y_{2}, \ldots\right.$, $\left.y_{j}, \ldots, y_{m}\right)$, respectively. The set of the corresponding elements of the weight matrix is $\left(w_{11}, w_{12}, \ldots, w_{i j}, \ldots, w_{n m}\right)$. The idea of back propagation is used to find the errors of node outputs in each layer. Without any loss of generality, the detailed learning processes of single layers for clarity are derived as follows. The derivation can be extended easily to the multipleoutput case. The goal of the proposed learning algorithm is to minimise the least-squares error function

$$
E=\frac{1}{2}\left(T_{j}-O_{j}\right)^{2}
$$

where $O_{j}=\max \left(\min \left(x_{i}, w_{i j}\right)\right), T_{j}$ is the desired FNN output value, $O_{j}$ is the actual value, and the least-squares error between them is $E$. The general parameter learning rule used is

$$
\frac{\partial E}{\partial w_{i j}}=\frac{\partial E}{\partial O_{j}} \cdot \frac{\partial \mathrm{O}_{j}}{\partial \mathrm{w}_{i j}}
$$

where

$$
\frac{\partial O_{j}}{\partial w_{i j}}=\frac{\left.\partial \bigvee\left(x_{i}, w_{i j}\right)\right)}{\partial \bigwedge\left(x_{s}, w_{s j}\right)} \frac{\partial \bigwedge\left(x_{s}, w_{s j}\right)}{\partial w_{s j}}
$$

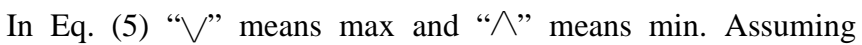
$\partial O_{j} / \partial w_{i j}=\Delta$ and $-\partial E / \partial O_{j}=\delta_{j}$, the changes of the weight will be obtained from a $\delta$-rule as follows:

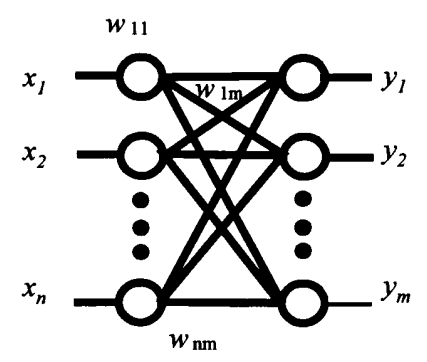

Fig. 3. FNN net topology. 


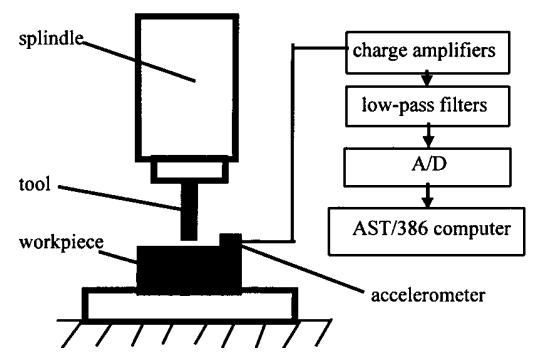

Fig. 4. Schematic diagram of the experimental set-up.

$$
\Delta w_{i j}=\mu \delta_{j} \Delta
$$

where $\mu$ is the learning rate, $\mu \in[0,1]$. Detailed learning algorithms are shown in [6].

\section{Experimental Set-up and Results}

\subsection{Experimental Set-up}

Drilling tests were performed on a machining centre MakinoFNC74-A20. A schematic diagram of the experimental set-up is shown in Fig. 4. During the experiments, the vibration signal is measured by means of an accelerometer mounted on the workpiece. The vibration signal is first passed through charge amplifiers (PS-506) and low-pass filters (4 kHz) and is then sampled by an A/D (wave form analyser AF-550A, sample frequency $25 \mathrm{kHz}$ ), then is input to a personal computer (AST/386). Drilling experiments were carried out on the machine $M C$ using a 3-9 $\mathrm{mm}$ diameter high-speed-steel twist drill and a $40 \mathrm{Cr}$ workpiece. All the experiments were carried out under dry conditions without coolant, but the method is also suitable for use with a coolant.

\subsection{Tool States}

The tool wear condition was divided into five states, i.e. initial wear, normal wear, acceptable wear, severe wear and failure $[7,8]$. Based on the flank wear of the tool, these conditions are summarised in Table 1.

The fuzzy membership functions of drilling conditions based on experimental data and the observed system behaviour are set for output indices of the FNN, which are the fuzzy membership functions of the drilling condition and are shown in Fig. 5. The reason for choosing a trapezoid shape is that it is difficult to quantify what exact percentage of the tool condition corre-

Table 1. Tool condition classification.

\begin{tabular}{ll}
\hline Tool condition & Flank wear \\
\hline Initial wear & $0<$ wear $<0.1 \mathrm{~mm}$ \\
Normal wear & $0.05<$ wear $<0.3 \mathrm{~mm}$ \\
Acceptable wear & $0.25<$ wear $<0.5 \mathrm{~mm}$ \\
Severe wear & $0.45<$ wear $<0.6 \mathrm{~mm}$ \\
Failure & ---- \\
\hline
\end{tabular}

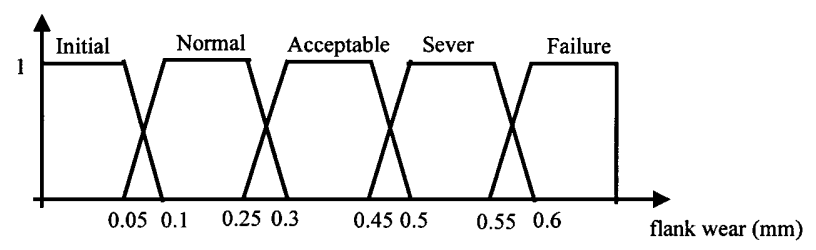

Fig. 5. Fuzzy membership function of drilling conditions.

sponds to a certain linguistic variable. In order to improve the training speed of the FNN, the tool wear conditions are coded as follows: initial $(1,0,0,0,0)$; normal $(0,1,0,0,0)$; acceptable $(0,0,1,0,0)$; severe $(0,0,0,1,0)$; and failure $(0,0,0,0,1)$. If the tool condition is normal, the output values of the FNN are $(0,1,0,0,0)$.

\subsection{Monitoring Indices}

Based on the cutting vibration signal, the mean value of each frequency band was used to describe the characteristics of different tool conditions, as shown in Fig. 6. The indices selected are summarised as follows:

$$
\begin{aligned}
x_{1}= & \text { the r.m.s. of the signal in the frequency band } \\
& {[0,300] \mathrm{Hz} } \\
x_{2}= & \text { the r.m.s. of the signal in the frequency band } \\
& {[300,600] \mathrm{Hz} } \\
x_{3}= & \text { the r.m.s. of the signal in the frequency band } \\
& {[600,1000] \mathrm{Hz} } \\
x_{4}= & \text { the r.m.s. of the signal in the frequency band } \\
& {[1000,1500] \mathrm{Hz} } \\
x_{5}= & \text { the r.m.s. of the signal in the frequency band } \\
& {[1500,2500] \mathrm{Hz} }
\end{aligned}
$$

The above parameters are the input values of the FNN.

\subsection{Experimental Results}

A total of 30 cutting tests were conducted under various cutting conditions. Twenty samples were randomly picked as learning samples. The samples remaining were used as the test samples in the classification phase. The final tool condition decision is made according to:

$$
J=\max \left(y_{i}\right) \quad(i=1,2,3,4,5)
$$

where $y_{i}$ is the output value of the trained FNN. The maximum value of the $y_{i}$, namely $J$, is converted to 1 , and the others are converted to 0 . For instance, if $J=y_{2}=0.8$, the output of FNN is $(0,1,0,0,0)$, the tool belongs to the normal wear condition. The test results are shown in Table 2 . It shows that the results meet the needs of the application.

\section{Conclusions}

In a manufacturing system, machining efficiency is greatly influenced by the tool condition in the cutting process. One of the most complex problems in tool condition monitoring is that of describing, with sufficient accuracy, the relationship 
(a)

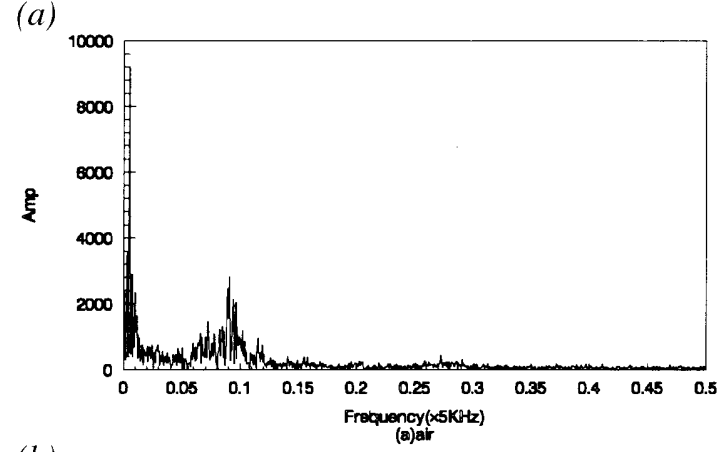

(b)

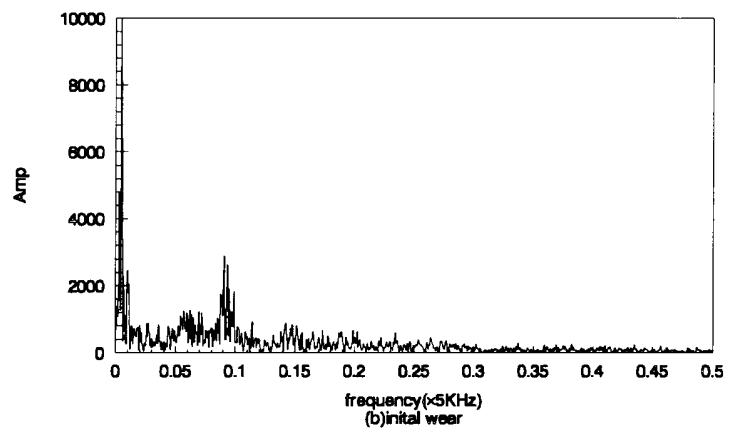

(c)

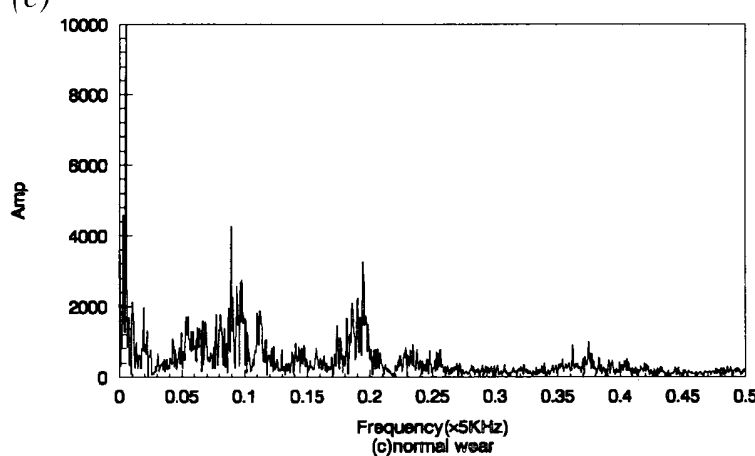

(d)

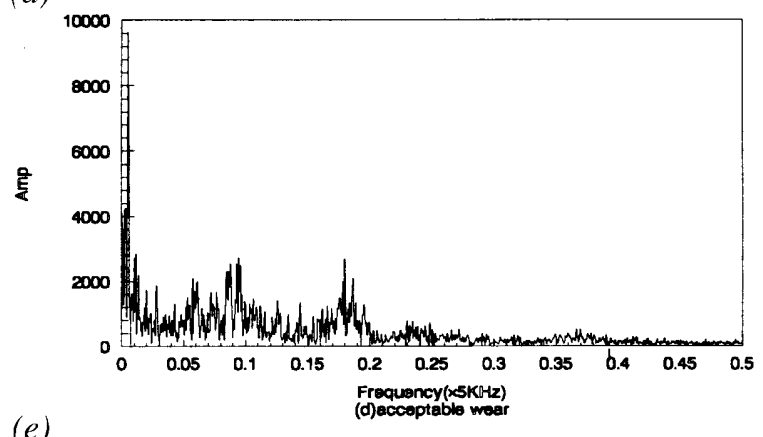

(e)
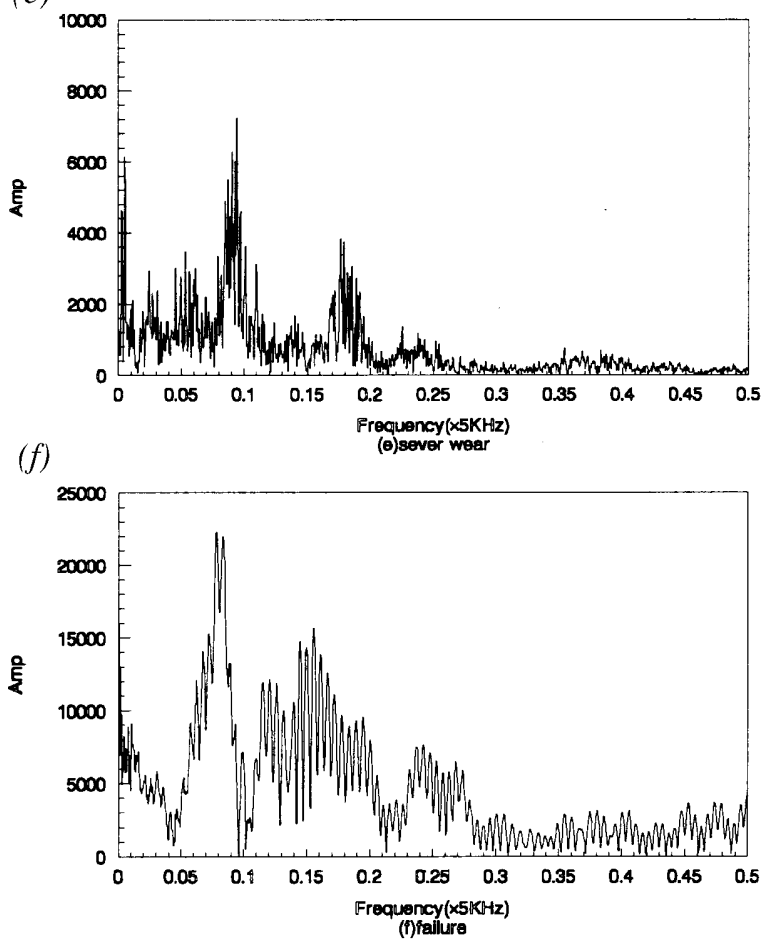

Fig. 6. Vibration spectral (FFT). Cutting speed: $380 \mathrm{rev} \mathrm{min}^{-1}$; feed: $20 \mathrm{~mm} \mathrm{~min}^{-1}$; work material: $40 \mathrm{Cr}$ steel; tool: high-speed-steel twist.

Table 2. Test results.

\begin{tabular}{lc}
\hline Tool condition & Recognition rate $(\%)$ \\
\hline Air cutting & 100 \\
Initial & 52 \\
Normal & 82 \\
Acceptable & 70 \\
Severe & 68 \\
Failure & 100 \\
\hline
\end{tabular}

between the tool wear condition and the features of the measured signal under a given cutting condition. In this paper, a new FNN is introduced for tool condition monitoring in machining. The following conclusions can be drawn from the investigation:
1. The fuzzy relationship between the tool condition and monitoring indices may be identified by using a fuzzy neural network

2. The training of the FNN is faster than for a back propagation-type neural network (BP).

3. The r.m.s. of the frequency bands increases as the flank wear increases.

It has also been shown that the coupling between the tool wear condition and the vibration signal being measured is adequate.

\section{References}

1. T. Nakajima, J. Ahn and T. Sata, "Tool breakage monitoring by means of fluctuations in spindle rotational speed", Annals CIRP, 36(1), pp. 49-52, 1987.

2. L. C. Lee, K. S. Lee and C. S. Gan, "On the correlation between dynamic cutting force and tool wear", International 
Journal of Machine Tools and Manufacture, 29(3), pp. 295303, 1989.

3. S. B. Rao, "Metal cutting machine tool design - a review", Journal of Manufacturing Science and Engineering", Transactions ASME, 119(4), pp. 713-716, 1997.

4. A. A. Houshmand, G. D. Herrin and E. Kannatey-Asibu, "Dynamic model for tool wear detection using acoustic emission", Control of Manufacturing Processes, American Society of Mechanical Engineers, Dynamic Systems and Control Division (Publication) DSC 28, pp. 13-22.

5. K. Danai and A. G. Ulsoy, "Dynamic state model for on-line tool wear estimation in turning", Journal of Engineering for Industry, Transactions ASME, 109(4), pp. 396-399, 1987.

6. Xiaoli Li, Yingxue Yao and Zhegun Yuan, "On-line tool condition monitoring system with wavelet fuzzy neural network", Journal of Intelligent Manufacture, 8(4), pp. 271-276, 1997.

7. F. R. Biglari and X. D. Fang, "Real-time fuzzy logic control for maximising the tool life of small-diameter drills", Fuzzy Sets and Systems, 72(1), pp. 91-101, 1995.

8. A. Blanco, M. Delgado and I. Requena, "Identification of fuzzy relational equations by fuzzy neural networks", Fuzzy Sets and Systems, 71, pp. 215-226, 1995. 